\title{
Empirical examination of the ability of children to consent to clinical research
}

Nancy Ondrusek, Rona Abramovitch, Paul Pencharz and Gideon Koren The Hospital for Sick Children, Toronto and the University of Toronto

\section{Abstract}

This study examined the quality of children's assent to a clinical trial. In subjects younger than 9 years of age, understanding of most aspects of the study was found to be poor to non-existent. Understanding of procedures was poor in almost all subjects. In addition, voluntariness may have been compromised in many subjects by their belief that failure to complete the study would displease others.

If the fact that a child's assent has been obtained is used to justify the exposure of that child to the potential harm of a non-therapeutic blood sample, the assent must be meaningful. In the nutrition study observed here, the quality of the assent of children younger than 9 years of age was very poor. The assent therefore did not provide a valid justification for requesting a blood sample from these children. This study indicates that most children younger than 9 years of age cannot be expected to consent or assent to clinical research in a meaningful way. The current age of 7 years for initiating assent (in addition to parental consent) is possibly not appropriate and should be reconsidered.

(Fournal of Medical Ethics 1998;24:158-165)

Keywords: Consent; children; research

While there is some information about children's capacity to assent to research, there is almost no information about the extent to which that capacity is realised amongst the thousands of children across Canada who assent to medical research every year. Abramovitch et al examined children's understanding in a research setting, but the research in which the subjects took part were psychological studies with very simple designs. ${ }^{12}$ Whether the findings from these studies could hold for children participating in complex medical studies involving numerous procedures and purposes involving specialised medical concepts is not known. The few studies which have looked at understanding amongst children participating in actual medical research were complicated by the fact that subjects were also patients and the studies were related to the treatment of their illness, so that previous knowledge and experience may have affected their understanding. ${ }^{34}$ In addition, Lewis's study, the abilities of groups of children, rather than individuals, were examined. ${ }^{4}$

Furthermore, these studies did not consider children's understanding in the context of the disclosure process. Woodward's cholera stu\&gy showed that adults are capable of understandigg large amounts of complex medical information. ${ }^{5}$ Numerous other studies have shown, howev, that this capacity is usually not realised in routiane consent situations. It is hypothesised that routin assent situations similarly, neither facilitate unde्eक standing nor maximise voluntariness.

The present pilot study was designed measure the quality of assent to a non-therapeutec clinical trial. It was hoped that by examining understanding and experience amongst childr⿳⺈ participating in an actual medical study, some information could be gained about the strengths and weaknesses of current assent practices. Sueh descriptive information could be used to genera荞e models for the assent process.

We focused on the assent process amongst healthy children participating in a notherapeutic study. It was hoped that by examining understanding in children who were not routine exposed to the medical system, the effects of the assent process on understanding and the research experience could be better isolated. Furthermote it could be argued that understanding of chldren's ability to assent is of particular importanfs for non-therapeutic research, where compromises to a child's autonomy are not balanced by potew tial direct health benefits.

To date, the question of whether or not it appropriate to subject children to blood sampliffg for the sake of research has been based on conceptual analysis. To our knowledge this is the first time that the assent process has be $\bar{n}$ examined in healthy children volunteering to pro vide a blood sample for research.

\section{Methods}

The design of this study was a cross-sectional sievey. The instrument was a semi-structured interview, administered by the author. 


\section{(A) SUBJECTS}

Subjects were eighteen children, 5 to 18 years old, who had agreed to participate in a study in the Division of Clinical Nutrition, The Hospital for Sick Children. Every individual who participated in the nutrition study was included in the consent study. Subjects were recruited through various forms of advertisement. Of the eighteen subjects, four responded to a newsletter, nine to posters and five to letters.

\section{(B) PROCEDURE}

Upon arrival in the Division of Clinical Nutrition, the prospective subjects were introduced to and informed of the roles of the primary investigator and research nurse for the nutrition study, and the primary investigator of the present consent study. Prospective subjects were then informed about the details of the study, using a standardised clinical information form, shown in Appendix 1. The form also contained information about the consent study.

It should be noted that, with the information sheet serving as the primary source of information about the study, the quality of the disclosure was a limiting factor in participants' ability to understand the study. Thus, rather than providing some objective measure of children's overall capacity, the results reveal only the subjects' performance under the conditions of this study.

Children 13 years of age and over were given the form to read for themselves. The primary investigator of the nutrition study read the information form to those in the 12 and under age group. Any questions raised by prospective subjects or their parents were answered by the primary investigator of the nutrition study. Following disclosure, written consent of parents and verbal assent of children under sixteen were obtained. Children 16 years of age and over were permitted to consent for themselves.

\section{Nutrition study}

The purpose of the nutrition study was to obtain normal values for body compartment (ie muscle, fat and water) measurements for children ranging in age from 5 to 18. Participants arrived on the morning of the study after fasting overnight and were subjected to a variety of tests. Two versions of the study were offered, short and long (see below). Before coming to the hospital, children 16 years of age and over, and parents of children less than 16 years of age, had been given a brief description of the procedures over the telephone by the primary investigator of the nutrition study. Subjects chose which version of the study they would undertake. The difference between the full study and the shorter, less invasive version, was that in the latter, the two blood tests, and the drinking of the sodium bromide water were omitted. Time taken to complete the long or short versions of the study was approximately four and two hours, respectively.

Of the eighteen subjects who participated, five completed the shorter version. In all cases, this decision was made before coming to the hospital for the study, and the description of the procedures altered accordingly. For children under 16, because the decision about which version of the study would be completed was made before subjects arrived for the study, it is not known whether the decision was largely the child's or the parent(s)', or what factors led to each choice.

Following completion of the nutrition study, subjects were given $\$ 20$ if they had participated in the shorter study, and $\$ 40$ if they had completed the longer version.

\section{INTERVIEWS}

Following disclosure and consent to the nutrition study, subjects were asked if they would agree to participate in the present study and verbal consent and assent were obtained. Each subject was interviewed privately by the author. In the hopes of maximising candidness it was stressed to the subjects that the interviewer was not part of the nutrition study, and that all responses would remain confidential.

\section{QUESTIONNAIRE}

Participants were questioned using a semistructured interview, shown in Appendix II. The interview was given in two parts, with eight questions given before participation in the nutrition study, and eight questions following completion of the nutrition study. Each set of questions took approximately five minutes. The questions asked before the study examined subject's knowledge about the content of the study, namely, the purpose, procedure, and potential harms and benefits. Questions were also asked about the subject's previous experience regarding needles and blood sampling. Questions after the study examined subjects' knowledge of and feelings about the right to withdraw. Subjects were also asked if they were prepared for what happened, what had been good and bad about the study, and why they had agreed to participate.

Interviews from previous studies ${ }^{12}$ were used to guide the wording of the questions. Questions about experience were asked with the intention of providing a source of internal validity; in other 
words, how apparent understanding compared with the actual experience.

\section{(c) Coding the data}

Data were sorted into descriptive categories. Categories were created from the responses. Once categories had been created, the responses were sorted. To validate the coding system, a second scorer independently sorted the responses using the established categories.

\section{Results}

All subjects who participated in the nutrition study agreed to participate in the consent study. Because this was a pilot study, and there were only eighteen subjects representing a broad range (ages 5 to 18), it was not reasonable to divide results into sub-groups. The data for each individual are therefore presented separately. Each available subject was interviewed, and no comparison groups were defined at the outset of the study. A preliminary review of the data suggested that for some elements of the consent process, understanding improved markedly around 9 years of age. Where relevant, the differences in responses between younger and older children will be noted.

\section{(A) UNDERSTANDING OF CONTENT OF THE STUDY}

The first four questions in the interview probed subjects' knowledge of the content of the study, that is, the purpose, procedures, and potential harms and benefits to the subject and to others.

\section{Purpose of the study}

The clinical information sheet served as the source from which subjects were expected to draw their answers. The explanation of purpose was as follows: "What we eat affects the amounts of muscle, fat and water in our body. The amounts also can change as we get older or when we get sick. We want to learn more about these changes by measuring the number of calories that you need, as well as the amount of muscle, fat and water there is in a healthy person like yourself... . With all of the information we will be able to tell you how many calories you need to eat and how much muscle and fat you have. Also, this information will help us treat sick children."

Subjects' responses to the question related to the purpose of the study fell into four basic categories: "I don't know"; "for a check-up" or other purposes related to their own health; "to measure calories/muscle/fat"; and "to help doctors/sick kids". In some cases more than one response was given. Overall, understanding of purpose was poor. A definite age-related pattern is apparent in the understanding of the purpose. 过 children under 9 years of age either could not 9 any way describe the purpose, or they believed the study was in some way related to checking the fir own health. With the exception of one 13-yearold, all subjects over 9 years of age responded that the purpose of the study was to measure son aspect of the body such as calories or fat, or the the study would in some way help doctors/sick children.

Only four of the eighteen subjects listed both measurement of calories, etc and helping docto sick children. Even these subjects, however, lack $\overrightarrow{\mathrm{ed}}$ a thorough understanding of the scientific put pose. This is demonstrated by the fact that none of the subjects could make a clear link between the measurement of their bodies and the way thege measurements would be used to help sigk children. As a matter of fact, one 18-year-old reał ised that he did not understand the purpose, when he responded "it [the information form] says it̄s [the study] supposed to help kids, but I'm not sure how". All adult subjects responded that the purpose was to measure some aspect of the body or generally to help doctors/sick children.

\section{Procedures}

Unlike understanding of the purpose, ability to 1 要 the procedures involved in the nutrition study did not show an age-related pattern.

Scoring was based on the number of correet procedures mentioned. Children were given of point for each correct item named and lost ore point for mentioning a procedure which was nøt part of the study. The total possible number off correct answers was four for the short version and six for the long version of the nutrition study. TRe final score for each subject was the total of correct answers, minus the total of incorrect answers, divided by the total possible number of corret answers, expressed as a percentage (correct incorrect / total $\times 100 \%$ ).

In response to the question "What is going to happen to you?", the overall mean score was $46.5 \%$. With the exception of the two youngesst subjects, who both scored $0 \%$ correct, age was not an important factor in determining score. though the overall score was poor, several of the procedures were listed by most of the subjects. Qf those having blood taken, only one 6-year-old afd one subject from the adult control group failed mention this test.

Some subjects listed procedures that were nt part of the nutrition study. Four subjects, rangi\&g in age from 11 to 17 , mentioned measuring कf heart rate, and one 14-year-old thought that of $x$-ray was part of the study. Again, results from the 
adult group were similar, with three of the subjects incorrectly naming heart rate or electroencephalograph (ECG) as one of the study procedures.

\section{Potential harms}

In addition to being informed of what procedures are involved in a research study, subjects must be informed of the potential harms that may accompany their participation. Response to the question "Is anything bad going to happen to you because you are in this study?", were categorised descriptively. Those children who responded "nothing" to the first question, were given the additional probe "Is there anything that you might not like?" Again, responses were divided into those from subjects having and not having blood tests.

All but one of the children over 9 years of age mentioned blood taking when asked about potential harms, whereas only one of the five children 9 years old or younger mentioned this harm. All but one of the five children not having blood taken gave the "correct" answer that there was no risk to this study, with one child falsely believing he would be having blood taken.

All but two of the subjects who mentioned blood taking or needles as a potential harm were concerned with the pain or unpleasantness of venipuncture. The two oldest subjects, however, mentioned contracting AIDS as a possible risk. It is notable that, with the exception of the mention of AIDS, and the risk of "getting electrocuted" also mentioned by one of the 18-year-olds, the only harm that was mentioned was the discomfort of having blood taken, which was also the only harm described in the information sheet. This finding is even more interesting when one considers that the question regarding potential harm was posed twice, once as "possible bad things?" and, to those who responded "nothing", a second time as "anything you might not like?" The implications of this observation to the disclosure process will be discussed below.

\section{Benefits}

Subjects were also probed to determine their beliefs about the benefits, both to themselves and to others, that might result from their participation in the nutrition study. In response to the question "What good things might happen to you because you are in this study" a range of answers was given, with "nothing", expressed by five of the subjects, as the most common response. Given that this study was "non-therapeutic", in the strictest sense "nothing" is the "correct" response to the question of individual benefit. With the exception of one 7-year-old, this answer came from children 14 years of age and over.
Three children, aged 5, 8, and 9, gave "don't know" in response to the question of personal benefit. The feeling that they would benefit by having "fun", was expressed by three children, aged 7,7 , and 9 . Three subjects aged 8,14 and 17 mentioned "knowing about calories and/or how much muscle they have" as a direct benefit of participation.

Again the responses from subjects over 9 years of age can be distinguished from the responses of younger subjects. With two exceptions, the responses "don't know" and "fun" came from children under 9 years of age, whereas "nothing", and the more detailed "knowing about calories/ muscle" came from children over 9 years of age.

It could be argued that, since the children who participated in this study had been asked to give up their time and occasionally their comfort so that others might benefit, it was important for the subjects to be aware of their contribution. Of the 14 children who were asked "What good things might happen to other people because you are in this study", only one 7-year-old responded "nothing". The majority of the children (eleven of the fourteen who were asked this question) did believe that their efforts would benefit others. Six of the eleven positive responders named the beneficiary of the study: two children identified doctors as benefiting, while four believed they were helping "sick children". The remaining five subjects who felt they were helping others did not specify whom they were helping. Since this latter group were not pressed for more information, no comment can be made as to whether this lack of specificity in the response reflects a true lack of knowledge or opinion on the part of the subjects. No age-related pattern was apparent in these responses.

\section{Right to withdraw}

Several of the questions asked after the subjects had completed the study examined their knowledge of, and confidence in, the rules of participation in medical research - the right to withdraw. In response to the question "Is it OK to stop", only two of the eight children under 10 years of age believed that it was acceptable to withdraw from the study, while seven of the nine children in the 10 and over age group were aware of this right.

But even amongst those who did state that it was permissible to stop, there appears to be a feeling of external influence which might prevent them from actually stopping. When asked how the investigator would feel if they withdrew from the study, twelve of the sixteen children who were asked this question thought that the investigator would respond negatively. Actual responses ranged from "sad" to "mad". One 9-year-old and 
one 18-year-old suggested the complexity of the situation in their responses with, "she said you didn't have to, [but] probably would have felt sad" and "[she would be] disappointed, but should understand", respectively. Both responses suggest an understanding of the conflict between what the situation should be (one is free to withdraw), and the natural inclination of the investigator to be disappointed when her interests (completing the study) are not served. No age-dependent pattern was apparent in the responses to this question.

Participants under 16 years of age were also asked "How would your parent(s) have felt if you had stopped"? The majority of children felt their parents would respond negatively if they withdrew.

\section{(B) SUBJECTS' EXPERIENCES OF, AND FEELINGS ABOUT, PARTICIPATION}

The remainder of the questions asked after completion of the nutrition study explored children's experiences and motivations regarding the study. In response to the question "During the study did you ever want to stop," only three children, all over 10 years of age, responded "yes". Two subjects, aged 14 and 18, had wanted to stop during blood taking and one 13-year-old explained that at noon he was ready to give up the fasting. When asked why they didn't withdraw from the study, the same reasoning - since they had already invested something in the study, they wanted to see it through (for example, “Once I started, doesn't matter. No use stopping once [the needle] is in")-was offered by all three subjects. None of the subjects suggested that they continued the study to avoid the displeasure of the investigator or their parent.

It should also be noted that one 8-year-old subject who had said he had not wanted to stop at any time, had started crying while put in a room alone for measurement of muscle mass, and was only able to continue after being comforted by his mother. This fortuitous information indicates that what a child says may not always reflect what he/she really feels.

\section{Preparedness}

To explore children's preparedness for the procedures to which they were subjected, they were asked "Was anything surprising, unexpected, or different than you thought it would be"? Two subjects each named two items in response to this question, thus there are a total of twenty responses from 18 subjects. The most common response was "nothing", given by seven subjects, five of whom were 14 years of age or older. The next most common response given by subjects ranging in age from 6 to 14, was that some aspect of t苗e blood sampling had been surprising. Two of the children in this group (aged 7 and 8), along wh one 17-year-old, also found that their experiences in the hood (necessary to measure breath in order to calculate calories needed) were not exactly ass they had expected. As for the "other" respons $\overline{\bar{\Phi}}$, one 8-year-old was surprised by "everything" (thays subject only listed one of the four possible items In the "procedures" question), and one 7-year-ofd was surprised by the fact that he did not "get needles" (this subject had also incorrectly named "needles" when asked about potential harms). $\overline{\overline{3}}$

Similarly, children were asked whether they hâd ever been scared during the study. Eleven of the eighteen, ranging in age from 5-18, said they had not been frightened at any time. Of these, ofe 17-year-old and one 18-year-old clarified that they had been "nervous", but "not actually scared". Of the seven subjects who had felt scared at some point, four had been frightened by the blood sampling. Three of the subjects in thiss group had also found some aspect of the blow sampling surprising or unexpected.

One potential argument for the view the children cannot give informed consent to partiespate in research is that they lack the life experienge with which to guide their decisions. With this क्षू mind, children had been asked before participation in the nutrition study about prior experienge with blood sampling. Of the six who had been surprised or frightened by the blood sampling, four had not only recalled having had a blood test in the past, but had been able to provide at least some description of what it had been like. The for this group, prior experience with blog $\mathrm{d}$ sampling was no guarantee of preparedness for similar procedure in the research setting.

\section{Worst and best experiences}

It is often the case that calculation of risk for given research protocol is based mainly on the physical invasiveness of the procedures involveds. In the case of children, venipuncture is seen many as invasive and its appropriateness in paediatric non-therapeutic research has recently beema subject of intense debate in this country. To a larg్ extent, however, the amount of harm (or benefit) resulting from any particular event, is subjectize and will vary with each individual.

There is essentially no empirical eviden⿳⺈, however, verifying speculations about childrems personal experiences as research subjects. As ${ }^{\circ}$ beginning step to explore this issue, subjects wege simply asked what were the worst and best parts the study. While "needles" or "blood" were the most common answers, given by six subjects 
ranging in age from 6 to 18 , eight of the subjects found some other procedure to be the "worst part". Four of the subjects responded that there was no "worst part" of the study. A range of ages was represented in each category of responses.

Similarly, the portion of the study perceived to be the "best part", varied widely among subjects, with no age-related pattern apparent.

\section{Motivation to participate}

In the case of research which offers no direct medical benefit to the subject, it is of interest to know why an individual agrees to participate. When the subjects are children, the question becomes even more important, since an understanding of children's motivation to participate is a significant part of addressing the question of whether or not children can be considered "volunteers".

In this study children were simply asked "Why did you say 'yes' to being in this study"? There were five basic responses: 1) "don't know" (five children, ages 6-14); 2) "money" (four children, ages 7-13); 3) "fun" (two subjects, ages 5 and 9); 4) "interested in medicine/science" (four children, ages 14-18); and 5) "to help sick kids/others" (three children, ages 9, 14, and 18).

\section{Discussion}

An important limitation of this study is the variability of the disclosure, the majority of which is attributable to two factors: 1) older children read the form, while younger children had it read to them; and 2) extra explanation may or may not have been provided, depending on whether subjects or their parents asked questions. It should be stressed, however, that the purpose of this study was not to measure capacity, but to look at performance within a research setting. Thus the variability can be regarded as an observation of the assent process in this example of clinical research. Similarly, criticisms of the quality of the disclosure, constitute further observations about the assent process in this setting.

Questions asked before subjects took part in the study were designed to determine whether those who had agreed to take part understood what they had agreed to. Two interesting trends regarding understanding and age of participants emerged: 1) Understanding of several aspects of the study, namely: purpose, potential harms (for those taking part in the long study), right to withdraw, and potential benefits, showed a definite agerelated pattern, whereas understanding of other types of information, such as procedures, did not appear related to age. 2) For all of the areas where there was an age-related pattern in the response, 9 years of age seemed a changing point - the majority of those 9 years of age and younger did not understand those elements of the study, while the majority of those over 9 years of age did appear to understand the same elements.

The conclusions drawn from these results and the implications for research involving children depend on whether the poor understanding, amongst subjects less than 9 years of age, of purpose, potential harms, potential benefits and right to withdraw observed in this study reflect limitations created by external factors, such as the disclosure, or by developmental limitations.

The fact that older children were able to understand information that the younger children were not able to understand, and the fact that in all cases the change in understanding occurred at about 9 years of age, indicates that there is a developmental difference in understanding information. The results of other studies support the interpretation that there is a turning point in understanding at about 9 years of age. For example, Bauer, ${ }^{6}$ observed that children's understanding of health and illness changes dramatically around age 10. A change in understanding at the 9-11 age range has also been observed in ongoing studies of children's understanding of information forms (Abramovitch, personal communication). On the other hand, the findings about changes in understanding at age 9 do not tell us that children are incapable of understanding purpose, potential harms, potential benefits and right to withdraw. In fact, a number of studies have demonstrated understanding of purpose, potential harms, and right to withdraw, in children as young as 6 or 7 years of age. ${ }^{124}$

Voluntariness is one of the basic ethical requirements of participation in research. Our results suggest that for this group, there were significant influences which might have limited their freedom to withdraw. The knowledge of the right to withdraw showed a strong age effect, but may have been largely a function of the disclosure. Previous studies have demonstrated that even young children can understand that it is supposedly permissible to withdraw from participation. ${ }^{1}$

The results of this study suggest, however, that children's actual freedom to act on that knowledge may have been substantially limited. Almost all of the subjects, regardless of age, felt that the investigator would be unhappy if they withdrew once the study had started. It is possible that these beliefs about the feelings of those in authority would inhibit a child from withdrawing from a study. Assurance from the investigator that he would not be upset has been shown to affect children's 
stopping behaviour. ${ }^{2}$ On the other hand, assurance from parents that it was $O K$ not to participate did not affect participation rate in children $7-12$ years of age.

Although the question regarding motivation to participate was open-ended, only five different responses were given by the eighteen subjects. It is interesting that only three subjects claimed to be motivated by altruistic intentions, in light of the fact that the good feeling that results from helping others is often cited as an important psychological benefit of participation in non-therapeutic research. Of course, it cannot be concluded that helping others did not influence the decision of the others, but the fact that such a motivation was not mentioned by these latter fifteen subjects suggests that it was not the prime reason for agreeing to participate.

One explanation for this result may be that the majority of children are not yet capable of the abstract reasoning necessary for feelings of altruism. This explanation cannot be explored fully for these subjects, as there was no measure of abstract reasoning abilities. On the other hand, the majority of children aged 9 and over had indicated that their participation in the nutrition study would be of benefit to others. Furthermore, only one of the four subjects who were 17 years of age and over cited "helping others" as the reason for his participation.

\section{Prior experience and preparedness}

In light of a number of studies demonstrating that adequate preparation prior to a procedure can decrease anxiety in children, it might be expected that children who have actually experienced a procedure may find it less traumatic the second (or subsequent) time. For the children in the present study, prior experience with blood sampling or needles did not guarantee an anxiety-free, or even a fully prepared experience. It may be, however, that without the previous experience these same subjects would have been even more anxious/poorly prepared.

In this study, information about children's perception of harm was obtained by asking children who participated in research what part of the study was "the worst". In this group, blood sampling was named, more than any other procedure, as the worst part of the research experience. This experience can be substantially changed in the future with the use of new local anaesthetic creams. The procedure named as worst second most often was being confined in the plastic hood. Because this procedure is non-invasive, and has no lasting physical effects on the subject, it is likely to be considered relatively benign by medical staff and research ethics boards; this is demonstrated by the fact that discomfort in the hood was not listed in the risks section of the approved cliniml information form. Although the sample size Is small, these results reveal a discomfort that wais found by the children who listed it, to be worse than blood sampling, yet it was not weighte्లd heavily enough to be described as a potential harfn in the information form.

In summary, this study examined the quality $\overrightarrow{\oplus f}$ children's assent to a clinical trial. In subjects younger than 9 years of age, understanding \& \& $_{\mathrm{f}}$ most aspects of the study was found to be poor non-existent. Understanding of procedures w:As poor in almost all subjects. In addition, volunta ness may have been compromised in many subjects by their belief that failure to complete the study would displease others.

If the fact that a child's assent has been obtained is used to justify the exposure of that child to the potential harm of a non-therapeutic blood sa ple, the assent must be meaningful. In the nutrition study observed here, the quality of tofe assent of children younger than 9 years of age was very poor. The assent therefore did not provideoa valid justification for requesting a blood samp from these children.

This study indicates that most children younger than 9 years of age cannot be expected to consent or assent to clinical research in a meaningful w. The current age of 7 years for initiating assent fin addition to parental consent) is probably appropriate and should be reconsidered.

Nancy Ondrusek, MSc, is a PhD student at the Insitute of Medical Sciences, University of Toronto. Rota Abramovitch, PhD, is Professor of Psychology at ine $^{2}$ University of Toronto. Paul Pencharz, MD, is Professor of Paediatrics and Nutritional Sciences at the University of Toronto. Gideon Koren, $M D$, is Professor

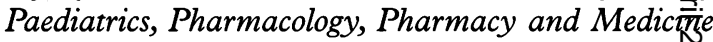
at the University of Toronto.

\section{References}

1 Abramovitch R, Freedman J, Thoden K, Nikolich C. Children's capacity to consent to participation in psychological researifh: empirical findings. Child Development 1991; 62:1100-9.

2 Abramovitch R, Freedman J, Henry K, Van Brunschot M. Children's capacity to agree to psychological research: knowledgeøf risk and benefits and voluntariness (submitted for publication, 1997).

3 Sussman E, Fletcher J. Participation in biomedical research: The consent process as viewed by children, adolescents, yo 10 adults, and physicians. Fournal of Pediatrics 1992;121,4: 547\$2.

4 Lewis C. A comparison of minors' and adults' pregnancy degisions. American fournal of Orthopsychiatry 1980;503:446-53.

5 Woodward W. Informed consent of volunteers: a direct measurement of comprehension and retention of information. Clinical Research 1979;27:248-52.

6 Bauer L. The development of the understanding of diabeter children with diabetes. PhD thesis: University of Toronto, 1990. 


\section{APPENDIX 1: Clinical information form, nutrition study}

This information sheet explains the study. After you read this we will ask you if you want to participate in this study. If you have any questions, please ask.

\section{CLINICAL INFORMATION SHEET}

What we eat affects the amounts of muscle, fat and water in our body. The amounts also can change as we get older or when we get sick. We want to learn more about these changes by measuring the number of calories that you need, as well as the amount of muscle, fat and water there is in a healthy person like yourself.

If you agree to be in our study, we will do some tests. These tests will take all morning. You will not be able to eat breakfast on the day of the study.

The first thing we will do is measure how tall you are, how much you weigh, and the thickness of fat we can pinch. A first blood sample (about $1 / 2$ teaspoon) will be taken. This involves being poked with a needle. Next we will give you some salty water to drink. After three hours we will take one more blood sample ( $1 / 2$ teaspoon).

Next we will measure the number of calories you need to eat each day. We do that by collecting your breath for one hour. You will have to lie on a bed with your head in a plastic hood. You will be able to watch TV or read a book during this time. Then we will take you to the Toronto General Hospital where you will be asked to lie on a bed for about 10 minutes while a machine measures the muscle in your body.

The last thing we will do is measure the amount of electricity in your body, using a special machine that is similar to the electrocadiogram machines that measure people's heartbeat. For this you will lie down on a bed for a few minutes, with four wires connecting you to the machine.

None of the measurements we will do will be bad for you. The only part that might hurt a bit is the two blood samples. With all of this infor- mation we will be able to tell you how many calories you need to eat and how much muscle and fat you have. Also, this information will help us treat sick children.

We will pay for your transportation costs to and from the hospital and lunch for you and your parents on the day that you are here for testing. If you have any further questions please call us.

\section{APPENDIX 2: Questionnaire, children's study}

Before medical study:

1 . Why is this study being done?

2. What is going to happen to you?

3 . What things are going to happen that you might not like?

4. What are the possible good things that might happen because you are in this study?

5 . Why did you say yes to being in this study?

6 . Why were you asked to be in this study?

7. Have you ever had a needle before? If yes, describe what it was like?

8 . Is there anything that you don't understand? After:

9. Did you know it was OK to stop if you didn't want to finish the study?

Do you think your mother would be upset if you stopped? Do you think Maria [the investigator] would be upset?

10. Once you started the study was there any time that you wanted to stop?

When? Why didn't you?

11. Did anything happen that you didn't expect?

12 . Were you ever scared?

13. Why did you say yes to being in this study?

14. What was the best part about being in this study?

15. What was the worst part?

16. If you had known what it would be like would you have said yes? Why? 